\title{
Adaptive pinning impulsive synchronization of dynamical networks with time-varying delay
}

\author{
Xiaoli Gong and Zhaoyan Wu*
}

*Correspondence:

zhywu@jxnu.edu.cn College of Mathematics and Information Science, Jiangxi Normal University, Ziyang Road, Nanchang, P.R. China

\section{Springer}

\begin{abstract}
In this paper, synchronization in dynamical networks with time-varying delay is investigated. Networks with time-varying coupling delay and node delay are both studied. By introducing adaptive strategy into pinning impulsive scheme, some effective and universal controllers are designed. In the proposed control schemes, for any given networks, the impulsive gains can adjust themselves to proper values when the impulsive intervals and some parameters are fixed. On the other hand, the impulsive instants can be estimated by solving a sequence of maximum value problems when the impulsive gains and some parameters are fixed. Based on the Lyapunov function method and mathematical analysis technique, several synchronization criteria are derived. Finally, numerical examples are performed to verify the effectiveness of the theoretical results.
\end{abstract}

Keywords: synchronization; dynamical network; pinning impulsive control; time-varying delay

\section{Introduction}

Dynamical networks consisting of nodes and edges are widely used to model the largescale real systems coupled with interactive individuals [1-3]. The nodes denote the individuals and the edges denote the interactions between a pair of interactive individuals. Synchronization, as a typical collective dynamical behavior of dynamical network, has drawn more and more attention from different fields [4-22]. Due to the complexity of dynamical networks, especially those dynamical networks coupling with chaotic systems, they cannot achieve synchronization themselves without external control. Therefore, how to design effective and low-cost controllers for achieving synchronization becomes an important and challenging issue.

In the impulsive control scheme, the controllers are added onto nodes only at some discrete instants, i.e., it is low-cost and easier to implement. Thus, an impulsive control scheme has been widely used to design controllers for achieving synchronization [9-19]. In [9], Sun et al. studied the synchronization of impulsively coupled complex networks. In [10], Yang et al. considered the exponential synchronization of uncertain delayed complex networks with nonidentical nodes and stochastic perturbations via hybrid adaptive and impulsive control. In [12], Deng et al. investigated the cluster synchronization of com-

(c) 2015 Gong and Wu. This article is distributed under the terms of the Creative Commons Attribution 4.0 International License (http://creativecommons.org/licenses/by/4.0/), which permits unrestricted use, distribution, and reproduction in any medium, provided you give appropriate credit to the original author(s) and the source, provide a link to the Creative Commons license, and indicate if changes were made. 
munity network via impulsive control. For any given dynamical network, one can choose proper impulsive gains and intervals such that the goal is realized.

As we know, many dynamical networks contain large number of nodes, which means that control of all nodes is high-cost and difficult to implement. Therefore, pinning control scheme, in which only a small fraction of nodes are controlled, has been widely adopted to design proper controllers combining with other control schemes [15-22]. Especially, in Refs. [17-19], stabilization and synchronization of dynamical networks are investigated by combining pinning and impulsive control, and some sufficient conditions are provided. From the sufficient conditions, for any given dynamical networks, one can easily estimate the impulsive gains and intervals for achieving the goals. However, different dynamical networks may have totally different system parameters and the number of controlled nodes may also be different, i.e., the pinning impulsive controllers with fixed impulsive gains and intervals are not universal. In Refs. [13-16], by introducing an adaptive strategy into (pinning) impulsive scheme, some adaptive (pinning) impulsive controllers are designed, which are universal to some extent. In Ref. [15], pinning impulsive synchronization of dynamical network without delay is investigated. However, time delays, including coupling delay and node delay, usually exist in many real networks. For example, the delays are usually time-varying in electronic implementation of analog networks due to the finite switching speed of amplifiers [17]. Therefore, pinning impulsive synchronization of dynamical network with delay, including node delay and coupling delay, deserves further studies.

Motivated by the above discussions, this paper investigates the synchronization of dynamical networks with time-varying delay via adaptive pinning impulsive control. Firstly, the dynamical network with time-varying coupling delay is considered and the corresponding controllers are designed. Secondly, the dynamical network with time-varying node delay is considered. According to the Lyapunov function method and mathematical analysis technique, the results are analytically proved. Compared with the obtained results in Refs. [15, 17-19], the main contributions of this paper are as follows: (1) effective and adaptive pinning impulsive controllers are designed for achieving synchronization of dynamical networks with time-varying delay, (2) the adaptive algorithms for not only the impulsive instants but also the impulsive gains are provided. That is, the obtained results extend those results obtained in Refs. [15, 17-19], to some extent.

The rest of this paper is organized as follows. In Section 2, the network models are introduced and some preliminaries are given. In Section 3, the adaptive pinning impulsive controllers for achieving synchronization are designed and the sufficient conditions are provided. In Section 4, several numerical simulations are performed to verify the results. In Section 5, the conclusion for this paper is given.

\section{Model description and preliminaries}

Consider a dynamical network consisting of $N$ nodes with time-varying coupling delay, which is described by

$$
\dot{x}_{i}(t)=f\left(x_{i}(t)\right)+c \sum_{j=1}^{N} a_{i j} H x_{j}(t-\tau(t)), \quad i=1,2, \ldots, N,
$$

where $x_{i}(t)=\left(x_{i 1}(t), x_{i 2}(t), \ldots, x_{i n}(t)\right)^{T} \in R^{n}$ is the state variable of node $i, f: R^{n} \rightarrow R^{n}$ is a nonlinear vector function, $c>0$ is the coupling strength, $H=\operatorname{diag}\left(h_{1}, h_{2}, \ldots, h_{n}\right)$ is the 
inner coupling matrix, $\tau(t) \geq 0$ is the time-varying delay. $A=\left(a_{i j}\right) \in R^{N \times N}$ is the zerorow-sum outer coupling matrix denoting the network topology and defined as follows: if there is a connection between node $j$ and $i(i \neq j)$, then $a_{i j} \neq 0$; otherwise, $a_{i j}=0$.

On the other hand, consider a dynamical network consisting of $N$ nodes with timevarying node delay, which is described by

$$
\dot{x}_{i}(t)=g\left(x_{i}(t-\tau(t))\right)+c \sum_{j=1}^{N} a_{i j} H x_{j}(t), \quad i=1,2, \ldots, N
$$

where $g: R^{n} \rightarrow R^{n}$ is a nonlinear vector function.

Networks (1) and (2) are said to achieve synchronization if $\lim _{t \rightarrow \infty}\left\|x_{i}(t)-s_{1}(t)\right\|=0$ and $\lim _{t \rightarrow \infty}\left\|x_{i}(t)-s_{2}(t)\right\|=0$, where $s_{1}(t)$ and $s_{2}(t)$ satisfy $\dot{s}_{1}(t)=f\left(s_{1}(t)\right)$ and $\dot{s}_{2}(t)=g\left(s_{2}(t-\right.$ $\tau(t)))$, respectively.

For achieving the synchronization, adaptive pinning impulsive controllers are designed and applied onto a small fraction of nodes in networks (1) and (2). The controlled networks are described by

$$
\begin{aligned}
& \dot{x}_{i}(t)=f\left(x_{i}(t)\right)+c \sum_{j=1}^{N} a_{i j} H x_{j}(t-\tau(t)), \quad t \neq t_{k}, \\
& x_{i}\left(t_{k}^{+}\right)=x_{i}\left(t_{k}^{-}\right)+b_{i}\left(t_{k}\right)\left(x_{i}\left(t_{k}^{-}\right)-s_{1}\left(t_{k}\right)\right), \quad t=t_{k},
\end{aligned}
$$

and

$$
\begin{aligned}
& \dot{x}_{i}(t)=g\left(x_{i}(t-\tau(t))\right)+c \sum_{j=1}^{N} a_{i j} H x_{j}(t), \quad t \neq t_{k}, \\
& x_{i}\left(t_{k}^{+}\right)=x_{i}\left(t_{k}^{-}\right)+b_{i}\left(t_{k}\right)\left(x_{i}\left(t_{k}^{-}\right)-s_{2}\left(t_{k}\right)\right), \quad t=t_{k},
\end{aligned}
$$

where $i=1,2, \ldots, N, k=1,2, \ldots$, the impulsive time instants $t_{k}$ satisfy $0=t_{0}<t_{1}<t_{2}<\cdots<$ $t_{k}<\cdots$, and $t_{k} \rightarrow \infty$ as $k \rightarrow \infty \cdot x_{i}\left(t_{k}^{+}\right)=\lim _{t \rightarrow t_{k}^{+}} x_{i}(t), x_{i}\left(t_{k}^{-}\right)=\lim _{t \rightarrow t_{k}^{-}} x_{i}(t)$. Any solution of (3) and (4) is assumed to be left continuous at each $t_{k}$, i.e., $x_{i}\left(t_{k}^{-}\right)=x_{i}\left(t_{k}\right) . b_{i}\left(t_{k}\right)$ is the impulsive gain at $t=t_{k}$ and $b_{i}(t)=0$ for $t \neq t_{k}$. Further, define $b_{i}\left(t_{k}\right)=b_{i}\left(t_{k}^{-}\right)=b_{i}\left(t_{k}^{+}\right)$.

Let $e_{k}^{(m)}(t)=x_{k}(t)-s_{m}(t)(m=1,2)$ be the synchronization errors, one can obtain the following error systems:

$$
\begin{aligned}
& \dot{e}_{i}^{(1)}(t)=f\left(x_{i}(t)\right)-f\left(s_{1}(t)\right)+c \sum_{j=1}^{N} a_{i j} H e_{j}^{(1)}(t-\tau(t)), \quad t \neq t_{k}, \\
& e_{i}^{(1)}\left(t_{k}^{+}\right)=e_{i}^{(1)}\left(t_{k}^{-}\right)+b_{i}\left(t_{k}\right) e_{i}^{(1)}\left(t_{k}^{-}\right), \quad t=t_{k},
\end{aligned}
$$

and

$$
\begin{aligned}
& \dot{e}_{i}^{(2)}(t)=g\left(x_{i}(t-\tau(t))\right)-g\left(s_{2}(t-\tau(t))\right)+c \sum_{j=1}^{N} a_{i j} H e_{j}^{(2)}(t), \quad t \neq t_{k}, \\
& e_{i}^{(2)}\left(t_{k}^{+}\right)=e_{i}^{(2)}\left(t_{k}^{-}\right)+b_{i}\left(t_{k}\right) e_{i}^{(2)}\left(t_{k}^{-}\right), \quad t=t_{k} .
\end{aligned}
$$


When $t=t_{k}$, arrange the synchronization errors $e_{i}^{(m)}(t)(m=1,2)$ according to their norms as follows:

$$
\left\|e_{i_{1}(t)}^{(m)}(t)\right\| \geq\left\|e_{i_{2}(t)}^{(m)}(t)\right\| \geq \cdots \geq\left\|e_{i_{\sigma}(t)}^{(m)}(t)\right\| \geq\left\|e_{i_{\sigma+1}(t)}^{(m)}(t)\right\| \geq \cdots \geq\left\|e_{i_{N}(t)}^{(m)}(t)\right\|
$$

where $i_{\sigma}(t) \in\{1,2, \ldots, N\}, \sigma=1,2, \ldots, N$, and if $\sigma \neq \kappa$, then $i_{\sigma}(t) \neq i_{\kappa}(t)$. Further, if $\left\|e_{i_{\sigma}(t)}(t)\right\|=\left\|e_{i_{\sigma+1}(t)}(t)\right\|$, then $i_{\sigma}(t)<i_{\sigma+1}(t)$. Let $P\left(t_{k}\right)=\left\{i_{1}\left(t_{k}\right), i_{2}\left(t_{k}\right), \ldots, i_{p}\left(t_{k}\right)\right\}$ be a set of $p$ nodes. If $i \in P\left(t_{k}\right)$, then $b_{i}\left(t_{k}\right)=b\left(t_{k}\right) \in(-2,-1) \cup(-1,0)$; otherwise, $b_{i}\left(t_{k}\right)=0$.

Assumption 1 Suppose that there exists a positive constant $L_{1}$ such that

$$
(y(t)-x(t))^{T}(f(y(t))-f(x(t))) \leq L_{1}(y(t)-x(t))^{T}(y(t)-x(t))
$$

holds for any $x(t), y(t) \in R^{n}$ and $t>0$.

Assumption 2 Suppose that there exist two positive constants $L_{2}$ and $L_{3}$ such that

$$
\begin{aligned}
(y(t) & -x(t))^{T}(g(y(t-\tau(t)))-g(x(t-\tau(t)))) \\
\leq & L_{2}(y(t)-x(t))^{T}(y(t)-x(t)) \\
& \quad+L_{3}(y(t-\tau(t))-x(t-\tau(t)))^{T}(y(t-\tau(t))-x(t-\tau(t)))
\end{aligned}
$$

holds for any $x(t), y(t) \in R^{n}$ and $t>0$.

Assumption 3 Suppose that the time-varying coupling delay $\tau(t)$ is differentiable and there exists a constant $\mu<1$ such that $\dot{\tau}(t) \leq \mu$.

\section{Main results}

Let $e^{(m)}(t)=\left(\left(e_{1}^{(m)}(t)\right)^{T},\left(e_{2}^{(m)}(t)\right)^{T}, \ldots,\left(e_{N}^{(m)}(t)\right)^{T}\right)^{T}(m=1,2), \tau_{k}=t_{k}-t_{k-1}$ be the impulsive intervals, $I$ be an identity matrix with appropriate dimension, $\lambda_{1}$ and $\lambda_{2}$ be the largest eigenvalues of $2 L_{1} I+c(A \otimes H)^{T}(A \otimes H)+c I /(1-\mu)$ and $2 L_{2} I+2 c(A \otimes H)+L_{3} I /(1-\mu)$, $\beta\left(t_{k}\right)=\left(1+b\left(t_{k}\right)\right)^{2}, \rho\left(t_{k}\right)=1-p\left(1-\beta\left(t_{k}\right)\right) / N$ for $t=t_{k}$ and $\rho(t)=1$ for $t \neq t_{k}$.

Theorem 1 Suppose that Assumptions 1 and 3 hold. If there exists a constant $\alpha>0$ such that

$$
\ln \rho\left(t_{k}\right)+\alpha+\widehat{L}\left(t_{k}\right) \tau_{k}<0, \quad k=1,2, \ldots
$$

holds, where $\widehat{L}(t)$ is the estimated value of $\lambda_{1}, \hat{L}(t)=\delta \sum_{i=1}^{N}\left(e_{i}^{(1)}(t)\right)^{T} e_{i}^{(1)}(t)$ with $\widehat{L}(0)>0$ and $\delta>0$ is the adaptive gain, then the synchronization of network (3) is achieved.

Proof Consider the following Lyapunov function:

$$
V(t)=\sum_{i=1}^{N}\left(e_{i}^{(1)}(t)\right)^{T} e_{i}^{(1)}(t)+\frac{\rho(t)}{2 \delta}\left(\widehat{L}(t)-\lambda_{1}\right)^{2}+\frac{c \rho(t)}{1-\mu} \int_{t-\tau(t)}^{t} \sum_{i=1}^{N}\left(e_{i}^{(1)}(\theta)\right)^{T} e_{i}^{(1)}(\theta) d \theta
$$

for $t \in\left(t_{k-1}, t_{k}\right], k=1,2, \ldots$ 
When $t \in\left(t_{k-1}, t_{k}\right)$, one has

$$
V(t)=\sum_{i=1}^{N}\left(e_{i}^{(1)}(t)\right)^{T} e_{i}^{(1)}(t)+\frac{1}{2 \delta}\left(\widehat{L}(t)-\lambda_{1}\right)^{2}+\frac{c}{1-\mu} \int_{t-\tau(t)}^{t} \sum_{i=1}^{N}\left(e_{i}^{(1)}(\theta)\right)^{T} e_{i}^{(1)}(\theta) d \theta
$$

and the derivative of $V(t)$ with respect to $t$ along the trajectory (5) is

$$
\begin{aligned}
\dot{V}(t)= & 2 \sum_{i=1}^{N}\left(e_{i}^{(1)}(t)\right)^{T} \dot{e}_{i}^{(1)}(t)+\frac{1}{\delta}(\widehat{L}(t)-\lambda) \widehat{\widehat{L}}(t)+\frac{c}{1-\mu} \sum_{i=1}^{N}\left(e_{i}^{(1)}(t)\right)^{T} e_{i}^{(1)}(t) \\
& -\frac{c(1-\dot{\tau}(t))}{1-\mu} \sum_{i=1}^{N}\left(e_{i}^{(1)}(t-\tau(t))\right)^{T} e_{i}^{(1)}(t-\tau(t)) \\
= & 2 \sum_{i=1}^{N}\left(e_{i}^{(1)}(t)\right)^{T}\left(f\left(x_{i}(t)\right)-f\left(s_{1}(t)\right)\right) \\
& +2 c \sum_{i=1}^{N} \sum_{j=1}^{N} a_{i j}\left(e_{i}^{(1)}(t)\right)^{T} H e_{j}^{(1)}(t-\tau(t)) \\
& +\left(\widehat{L}(t)-\lambda_{1}\right) \sum_{i=1}^{N}\left(e_{i}^{(1)}(t)\right)^{T} e_{i}^{(1)}(t)+\frac{c}{1-\mu} \sum_{i=1}^{N}\left(e_{i}^{(1)}(t)\right)^{T} e_{i}^{(1)}(t) \\
\leq & \widehat{L}(t) \sum_{i=1}^{N}\left(e_{i}^{(1)}(t)\right)^{T} e_{i}^{(1)}(t) . \\
& \frac{c(1-\dot{\tau}(t))}{1-\mu} \sum_{i=1}^{N}\left(e_{i}^{(1)}(t-\tau(t))\right)^{T} e_{i}^{(1)}(t-\tau(t)) \\
\leq & \left(e^{(1)}(t)\right)^{T}\left(2 L_{1} I+c(A \otimes H)^{T}(A \otimes H)+\frac{c I}{1-\mu}\right) e^{(1)}(t) \\
& \frac{c(\dot{\tau}(t)-\mu)}{1-\mu}\left(e^{(1)}(t-\tau(t))\right)^{T} e^{(1)}(t-\tau(t))+\left(\widehat{L}(t)-\lambda_{1}\right)\left(e^{(1)}(t)\right)^{T} e^{(1)}(t) \\
& \\
& \\
& \\
&
\end{aligned}
$$

According to the definition of $\widehat{L}(t)$, it is clear that $\widehat{L}(t)$ is a positive and monotone increasing function. Then one has

$$
\dot{V}(t) \leq \widehat{L}(t) V(t) \leq \widehat{L}\left(t_{k}\right) V(t)
$$

which gives

$$
V(t) \leq V\left(t_{k-1}\right) \exp \left(\widehat{L}\left(t_{k}\right)\left(t-t_{k-1}\right)\right), \quad t \in\left(t_{k-1}, t_{k}\right)
$$

Due to $x_{i}\left(t_{k}^{-}\right)=x_{i}\left(t_{k}\right)$ and $b_{i}\left(t_{k}\right)=b_{i}\left(t_{k}^{-}\right)=b_{i}\left(t_{k}^{+}\right), V(t)$ is left continuous at each $t_{k}$ according to the definitions of $\rho(t)$ and $V(t)$. Thus, when $t=t_{k}$, one has

$$
\begin{aligned}
V\left(t_{k}^{+}\right)= & \sum_{i=1}^{N}\left(e_{i}^{(1)}\left(t_{k}^{+}\right)\right)^{T} e_{i}^{(1)}\left(t_{k}^{+}\right)+\frac{\rho\left(t_{k}\right)}{2 \delta}\left(\widehat{L}\left(t_{k}\right)-\lambda_{1}\right)^{2} \\
& +\frac{c \rho\left(t_{k}\right)}{1-\mu} \int_{t_{k}-\tau\left(t_{k}\right)}^{t_{k}} \sum_{i=1}^{N}\left(e_{i}^{(1)}(\theta)\right)^{T} e_{i}^{(1)}(\theta) d \theta
\end{aligned}
$$




$$
\begin{aligned}
= & \left(1+b\left(t_{k}\right)\right)^{2} \sum_{i \in P\left(t_{k}\right)}\left(e_{i}^{(1)}\left(t_{k}^{-}\right)\right)^{T} e_{i}^{(1)}\left(t_{k}^{-}\right)+\sum_{i \notin P\left(t_{k}\right)}\left(e_{i}^{(1)}\left(t_{k}^{-}\right)\right)^{T} e_{i}^{(1)}\left(t_{k}^{-}\right) \\
& +\frac{\rho\left(t_{k}\right)}{2 \delta}\left(\widehat{L}\left(t_{k}\right)-\lambda_{1}\right)^{2}+\frac{c \rho\left(t_{k}\right)}{1-\mu} \int_{t_{k}-\tau\left(t_{k}\right)}^{t_{k}} \sum_{i=1}^{N}\left(e_{i}^{(1)}(\theta)\right)^{T} e_{i}^{(1)}(\theta) d \theta \\
= & \beta\left(t_{k}\right) \sum_{i=1}^{N}\left(e_{i}^{(1)}\left(t_{k}^{-}\right)\right)^{T} e_{i}^{(1)}\left(t_{k}^{-}\right)+\left(1-\beta\left(t_{k}\right)\right) \sum_{i \notin P\left(t_{k}\right)}\left(e_{i}^{(1)}\left(t_{k}^{-}\right)\right)^{T} e_{i}^{(1)}\left(t_{k}^{-}\right) \\
& +\frac{\rho\left(t_{k}\right)}{2 \delta}\left(\widehat{L}\left(t_{k}\right)-\lambda_{1}\right)^{2}+\frac{c \rho\left(t_{k}\right)}{1-\mu} \int_{t_{k}-\tau\left(t_{k}\right)}^{t_{k}} \sum_{i=1}^{N}\left(e_{i}^{(1)}(\theta)\right)^{T} e_{i}^{(1)}(\theta) d \theta .
\end{aligned}
$$

From the definition of $P\left(t_{k}\right)$, one has

$$
\frac{1}{N-p} \sum_{i \notin P\left(t_{k}\right)}\left(e_{i}^{(1)}\left(t_{k}^{-}\right)\right)^{T} e_{i}^{(1)}\left(t_{k}^{-}\right) \leq \frac{1}{N} \sum_{i=1}^{N}\left(e_{i}^{(1)}\left(t_{k}^{-}\right)\right)^{T} e_{i}^{(1)}\left(t_{k}^{-}\right),
$$

and

$$
\begin{aligned}
V\left(t_{k}^{+}\right) \leq & \beta\left(t_{k}\right) \sum_{i=1}^{N}\left(e_{i}^{(1)}\left(t_{k}^{-}\right)\right)^{T} e_{i}^{(1)}\left(t_{k}^{-}\right)+\frac{\rho\left(t_{k}\right)}{2 \delta}\left(\widehat{L}\left(t_{k}\right)-\lambda_{1}\right)^{2} \\
& +\frac{\left(1-\beta\left(t_{k}\right)\right)(N-p)}{N} \sum_{i=1}^{N}\left(e_{i}^{(1)}\left(t_{k}^{-}\right)\right)^{T} e_{i}^{(1)}\left(t_{k}^{-}\right) \\
& +\frac{c \rho\left(t_{k}\right)}{1-\mu} \int_{t_{k}-\tau\left(t_{k}\right)}^{t_{k}} \sum_{i=1}^{N}\left(e_{i}^{(1)}(\theta)\right)^{T} e_{i}^{(1)}(\theta) d \theta \\
= & \rho\left(t_{k}\right) V\left(t_{k}^{-}\right) .
\end{aligned}
$$

By mathematical induction, for any positive integer $k$, one has

$$
V\left(t_{k}^{+}\right) \leq V\left(t_{0}\right) \prod_{j=1}^{k} \rho\left(t_{j}\right) \exp \left(\widehat{L}\left(t_{j}\right)\left(t_{j}-t_{j-1}\right)\right), \quad k=1,2, \ldots
$$

If condition (7) holds, one has

$$
\rho\left(t_{j}\right) \exp \left(\widehat{L}\left(t_{j}\right)\left(t_{j}-t_{j-1}\right)\right) \leq \exp (-\alpha), \quad j=1,2, \ldots, k
$$

and

$$
V\left(t_{k}^{+}\right) \leq V\left(t_{0}\right) \exp (-k \alpha)
$$

which implies $V\left(t_{k}^{+}\right) \rightarrow 0$ for $k \rightarrow \infty$.

Then, for $t \in\left(t_{k}, t_{k+1}\right]$, one has

$$
V(t) \leq V\left(t_{k}^{+}\right) \exp \left(\widehat{L}\left(t_{k+1}\right)\left(t-t_{k}\right)\right)
$$

which implies $V(t) \rightarrow 0$ for $t \rightarrow \infty$, i.e., the error system (5) is stable about zero and the synchronization is achieved. This completes the proof. 
Theorem 2 Suppose that Assumptions 2 and 3 hold. If there exists a constant $\alpha>0$ such that

$$
\ln \rho\left(t_{k}\right)+\alpha+\widehat{L}\left(t_{k}\right) \tau_{k}<0, \quad k=1,2, \ldots
$$

holds, where $\widehat{L}(t)$ is the estimated value of $\lambda_{2}, \widehat{L}(t)=\delta \sum_{i=1}^{N}\left(e_{i}^{2}(t)\right)^{T} e_{i}^{2}(t)$ with $\widehat{L}(0)>0$ and $\delta>0$ is an adaptive gain, then the synchronization of network (4) is achieved.

Proof Consider the following Lyapunov function:

$$
\begin{aligned}
V(t)= & \sum_{i=1}^{N}\left(e_{i}^{(2)}(t)\right)^{T} e_{i}^{(2)}(t)+\frac{\rho(t)}{2 \delta}\left(\widehat{L}(t)-\lambda_{2}\right)^{2} \\
& +\frac{L_{3} \rho(t)}{1-\mu} \int_{t-\tau(t)}^{t} \sum_{i=1}^{N}\left(e_{i}^{(2)}(\theta)\right)^{T} e_{i}^{(2)}(\theta) d \theta
\end{aligned}
$$

for $t \in\left(t_{k-1}, t_{k}\right], k=1,2, \ldots$.

When $t \in\left(t_{k-1}, t_{k}\right)$, one has

$$
\begin{aligned}
V(t)= & \sum_{i=1}^{N}\left(e_{i}^{(2)}(t)\right)^{T} e_{i}^{(2)}(t)+\frac{1}{2 \delta}\left(\widehat{L}(t)-\lambda_{2}\right)^{2} \\
& +\frac{L_{3}}{1-\mu} \int_{t-\tau(t)}^{t} \sum_{i=1}^{N}\left(e_{i}^{(2)}(\theta)\right)^{T} e_{i}^{(2)}(\theta) d \theta,
\end{aligned}
$$

and the derivative of $V(t)$ with respect to $t$ along the trajectory (6) is

$$
\begin{aligned}
\dot{V}(t)= & 2 \sum_{i=1}^{N}\left(e_{i}^{(2)}(t)\right)^{T} \dot{e}_{i}^{(2)}(t)+\frac{1}{\delta}\left(\widehat{L}(t)-\lambda_{2}\right) \hat{\widehat{L}}(t)+\frac{L_{3}}{1-\mu} \sum_{i=1}^{N}\left(e_{i}^{(2)}(t)\right)^{T} e_{i}^{(2)}(t) \\
& -\frac{L_{3}(1-\dot{\tau}(t))}{1-\mu} \sum_{i=1}^{N}\left(e_{i}^{(2)}(t-\tau(t))\right)^{T} e_{i}^{(2)}(t-\tau(t)) \\
\leq & \left(e^{(2)}(t)\right)^{T}\left(2 L_{2} I+2 c(A \otimes H)+\frac{L_{3} I}{1-\mu}\right) e^{(2)}(t) \\
& +\frac{L_{3}(\dot{\tau}(t)-\mu)}{1-\mu}\left(e^{(2)}(t-\tau(t))\right)^{T} e^{(2)}(t-\tau(t)) \\
& +\left(\widehat{L}(t)-\lambda_{2}\right)\left(e^{(2)}(t)\right)^{T} e^{(2)}(t) \\
\leq & \widehat{L}(t) \sum_{i=1}^{N}\left(e_{i}^{(2)}(t)\right)^{T} e_{i}^{(2)}(t) \leq \widehat{L}\left(t_{k}\right) V(t)
\end{aligned}
$$

which gives

$$
V(t) \leq V\left(t_{k-1}\right) \exp \left(\widehat{L}(t)\left(t-t_{k-1}\right)\right), \quad t \in\left(t_{k-1}, t_{k}\right) .
$$


When $t=t_{k}$,

$$
\begin{aligned}
V\left(t_{k}^{+}\right)= & \sum_{i=1}^{N}\left(e_{i}^{(2)}\left(t_{k}^{+}\right)\right)^{T} e_{i}^{(2)}\left(t_{k}^{+}\right)+\frac{\rho\left(t_{k}\right)}{2 \delta}\left(\widehat{L}\left(t_{k}\right)-\lambda_{2}\right)^{2} \\
& +\frac{L_{3} \rho\left(t_{k}\right)}{1-\mu} \int_{t_{k}-\tau\left(t_{k}\right)}^{t_{k}} \sum_{i=1}^{N}\left(e_{i}^{(2)}(\theta)\right)^{T} e_{i}^{(2)}(\theta) d \theta \\
\leq & \rho\left(t_{k}\right) \sum_{i=1}^{N}\left(e_{i}^{(2)}\left(t_{k}^{-}\right)\right)^{T} e_{i}^{(2)}\left(t_{k}^{-}\right)+\frac{\rho\left(t_{k}\right)}{2 \delta}\left(\widehat{L}\left(t_{k}\right)-\lambda_{2}\right)^{2} \\
& +\frac{L_{3} \rho\left(t_{k}\right)}{2 \delta} \int_{t_{k}-\tau t_{k}}^{t_{k}} \sum_{i=1}^{N}\left(e_{i}^{(2)}(\theta)\right)^{T} e_{i}^{(2)}(\theta) d \theta \\
= & \rho\left(t_{k}\right) V\left(t_{k}^{-}\right) .
\end{aligned}
$$

Thus, similar to the proof of Theorem 1, the proof can be completed.

Remark 1 Generally, the impulsive interval $\tau_{k}$ is chosen as a constant $\tau_{0}$, i.e., $t_{k}-t_{k-1}=\tau_{0}$. Thus, for any given $\tau_{0}$ and $\alpha$, one can choose

$$
\begin{aligned}
& -\left(1-N\left(1-\exp \left(-\alpha-\widehat{L}\left(t_{k}\right) \tau_{k}\right)\right) / p\right)^{\frac{1}{2}}-1+\varepsilon \\
& \quad \leq b\left(t_{k}\right) \leq\left(1-N\left(1-\exp \left(-\alpha-\widehat{L}\left(t_{k}\right) \tau_{k}\right)\right) / p\right)^{\frac{1}{2}}-1-\varepsilon,
\end{aligned}
$$

such that conditions (7) and (8) hold, where $\varepsilon$ is an arbitrary small positive constant.

Remark 2 From conditions (7) and (8), if $p, N, b\left(t_{k}\right)$ and $\alpha$ are fixed, one can estimate the control instants $t_{k}$ through finding the maximum value of $t_{k}$ subject to $t_{k}<t_{k-1}-\left(\ln \rho\left(t_{k}\right)+\right.$ $\alpha) \widehat{L}^{-1}\left(t_{k}\right) / 2$ with $t_{0}=0, k=1,2, \ldots$.

Remark 3 Compared with the results in Ref. [15], this paper considers not only the dynamical network with time-varying coupling delay but also the one with time-varying node delay. Besides the adaptive algorithm for solving the impulsive instants, the algorithm for determining the impulsive gains is also given. Compared with the results in Refs. [17-19], this paper designs effective and adaptive controllers through introducing a proper adaptive strategy. From the derived conditions and proofs in Theorems 1 and 2, the largest eigenvalues $\lambda_{1}$ and $\lambda_{2}$ need not be calculated. That is, the obtained results in Refs. $[15,17-19]$ are extended to some extent.

\section{Numerical illustrations}

Example 1 Consider the synchronization of network (3) with time-varying coupling delay. The network topology is generated as the BA scale-free network [23] with $N=100$ and $m_{0}=m=3$. Choose the node dynamics as the Lorenz system [24]

$$
\begin{aligned}
& \dot{x}_{i 1}=10\left(x_{i 2}-x_{i 1}\right), \\
& \dot{x}_{i 2}=28 x_{i 1}-x_{i 1} x_{i 3}-x_{i 2}, \\
& \dot{x}_{i 3}=x_{i 1} x_{i 2}-8 / 3 x_{i 3},
\end{aligned}
$$




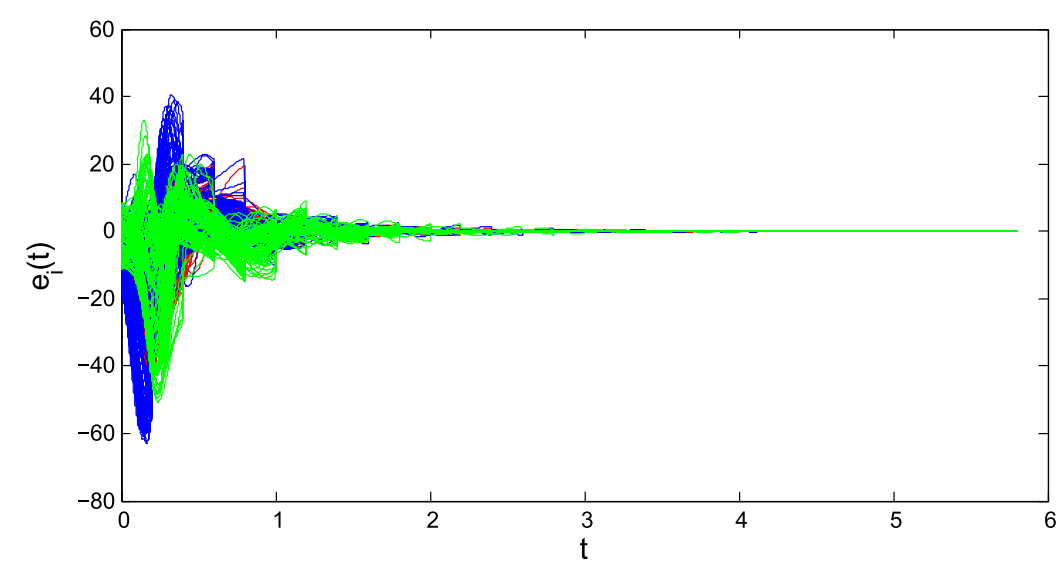

Figure 1 The orbits of $e_{i j}(t), i=1,2, \ldots, 100, j=1,2,3$. The red, blue and green lines denote the orbits of $e_{i 1}(t), e_{i 2}(t)$ and $e_{i 3}(t)$, respectively.

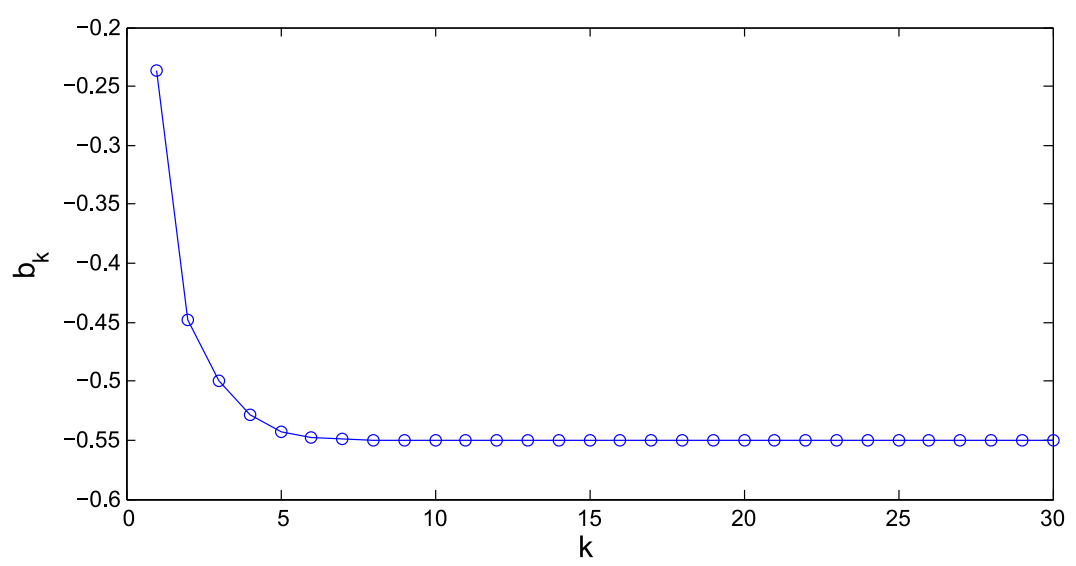

Figure 2 The orbit of $b\left(t_{k}\right)$ vs $k$.

the inner coupling matrix $H$ as an identity matrix and the time-varying delay as $\tau(t)=$ $0.2-0.2 \sin t$.

Firstly, choose $c=0.1$, the impulsive intervals $\tau_{k}$ as a constant $\tau_{0}=0.2, \alpha=0.001, \delta=$ $0.00002, \widehat{L}(0)=0.01$ and $p=20$. According to Remark 1 , choose $b\left(t_{k}\right)=(1-N(1-\exp (-\alpha-$ $\left.\left.\left.\widehat{L}\left(t_{k}\right) \tau_{k}\right)\right) / p\right)^{\frac{1}{2}}-1-\varepsilon$ with $\varepsilon=0.002$ such that condition (7) holds. Choose the initial values of $x_{i}(t)$ and $s(t)$ randomly. Figure 1 shows the orbits of synchronization errors $e_{i j}(t), i=$ $1,2, \ldots, 100, j=1,2,3$. Figure 2 shows the impulsive gain $b\left(t_{k}\right)$ versus $k$.

Secondly, choose $b\left(t_{k}\right)=-0.9, \alpha=0.001, p=20, \delta=0.00001, \widehat{L}(0)=0.01$ and the initial values of $x_{i}(t)$ and $s(t)$ randomly. The impulsive instants are estimated according to $\operatorname{Re}-$ mark 2 . Figure 3 shows the orbits of synchronization errors $e_{i j}(t), i=1,2, \ldots, 100, j=1,2,3$. Figure 4 shows the impulsive interval $\tau_{k}$ versus $k$.

Example 2 Consider the synchronization of network (4) with time-varying node delay. The network topology is also generated as the BA scale-free network [23] with $N=20$ and $m_{0}=m=3$, and the inner coupling matrix $H$ is chosen as an identity matrix. Choose the 


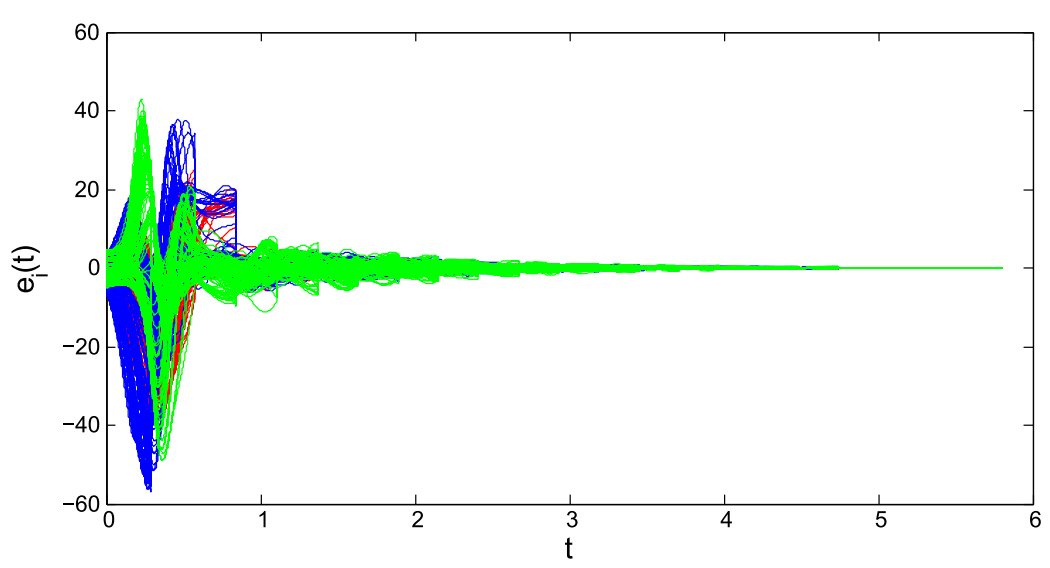

Figure 3 The orbits of $e_{i j}(t), i=1,2, \ldots, 100, j=1,2,3$. The red, blue and green lines denote the orbits of $e_{i 1}(t), e_{i 2}(t)$ and $e_{i 3}(t)$, respectively.

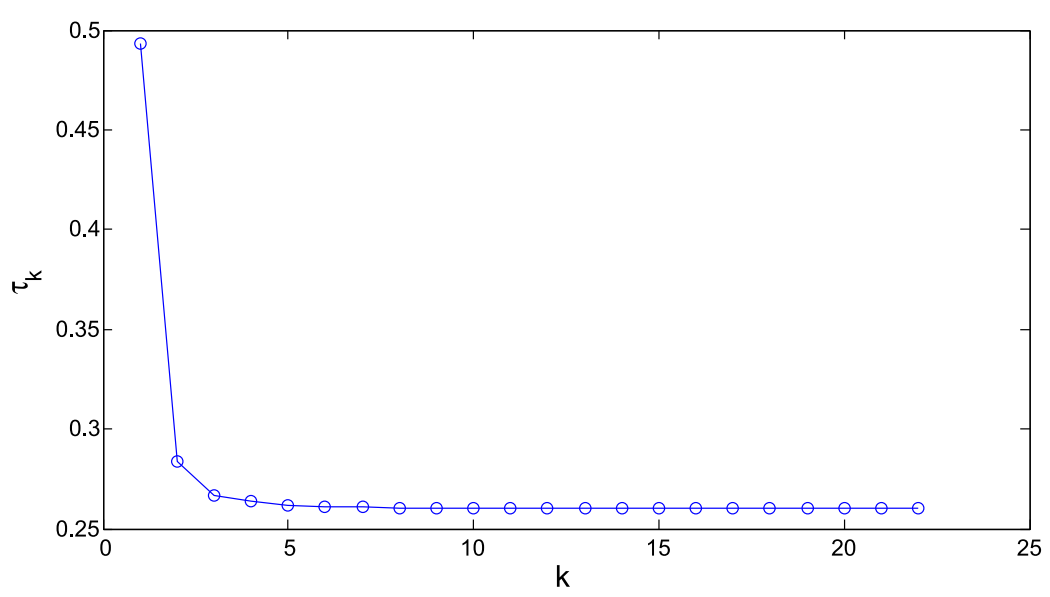

Figure 4 The orbit of $\tau_{k}$ vs $k$.

node dynamics as the delayed chaotic system [25]

$$
\dot{x}_{i}(t)=-x_{i}(t)+M_{1} f\left(x_{i}(t)\right)+M_{2} g\left(x_{i}(t-\tau(t))\right),
$$

where $x_{i}(t)=\left(x_{i 1}(t), x_{i 2}(t)\right)^{T}, f\left(x_{i}(t)\right)=g\left(x_{i}(t)\right)=\left(\tanh \left(x_{i 1}(t)\right), \tanh \left(x_{i 2}(t)\right)\right)^{T}, \tau(t)=\exp (t) /$ $(1+\exp (t))$ and

$$
M_{1}=\left[\begin{array}{cc}
1.8 & -0.1 \\
-2.5 & 0.3
\end{array}\right], \quad M_{2}=\left[\begin{array}{cc}
-1.6 & -0.6 \\
0.1 & -2.4
\end{array}\right] \text {. }
$$

Firstly, choose $c=0.1, \tau_{0}=0.5, \alpha=0.001, \delta=0.01, \widehat{L}(0)=0.01$ and $p=6$. According to Remark 1 , choose $b\left(t_{k}\right)=\left(1-N\left(1-\exp \left(-\alpha-\widehat{L}\left(t_{k}\right) \tau_{k}\right)\right) / p\right)^{\frac{1}{2}}-1-\varepsilon$ with $\varepsilon=0.002$ such that condition (7) holds. Choose the initial values of $x_{i}(t)$ and $s(t)$ randomly. Figure 5 shows the orbits of synchronization errors $e_{i j}(t), i=1,2, \ldots, 100, j=1,2$. Figure 6 shows the impulsive gain $b\left(t_{k}\right)$ versus $k$. 


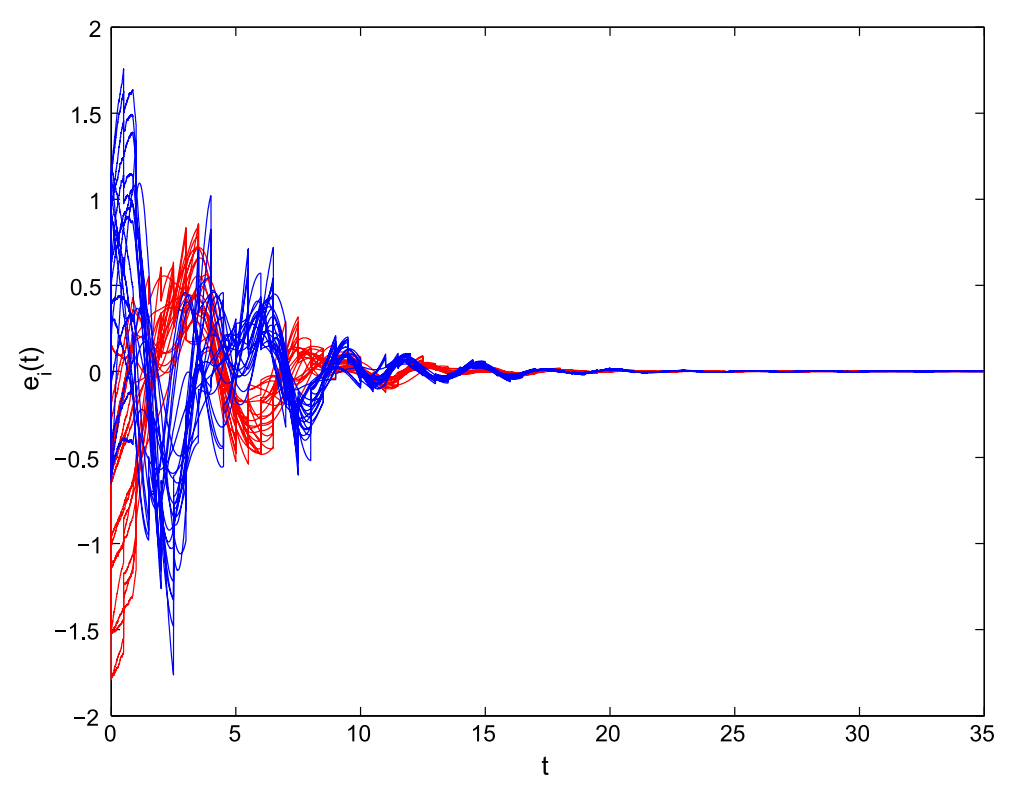

Figure 5 The orbits of $e_{i j}(t), i=1,2, \ldots, 20, j=1,2$. The red and blue lines denote the orbits of $e_{j 1}(t)$ and $e_{i 2}(t)$, respectively.

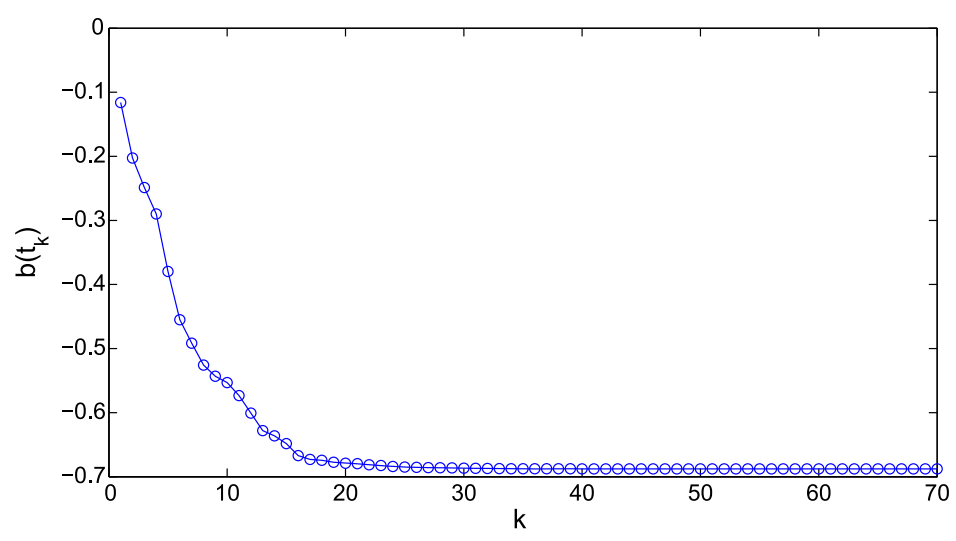

Figure 6 The orbit of $b\left(t_{k}\right)$ vs $k$.

Secondly, choose $b\left(t_{k}\right)=-0.9, \alpha=0.001, \delta=0.002, \widehat{L}(0)=0.01$, and the initial values of $x_{i}(t)$ and $s(t)$ randomly. The impulsive instants are estimated according to Remark 2 . Figure 7 shows the orbits of synchronization errors $e_{i j}(t), i=1,2, \ldots, 100, j=1,2$. Figure 8 shows the impulsive interval $\tau_{k}$ versus $k$.

\section{Conclusion}

In this paper, the synchronization of dynamical networks with time-varying delay is well studied via adaptive pinning impulsive control. Dynamical networks with both timevarying coupling delay and node delay are considered. Based on the Lyapunov function method and mathematical analysis technique, several sufficient conditions for achieving synchronization are derived. According to the discussions in Remarks 1 and 2, the impulsive gains or instants can adjust themselves to proper values or be estimated by solving 


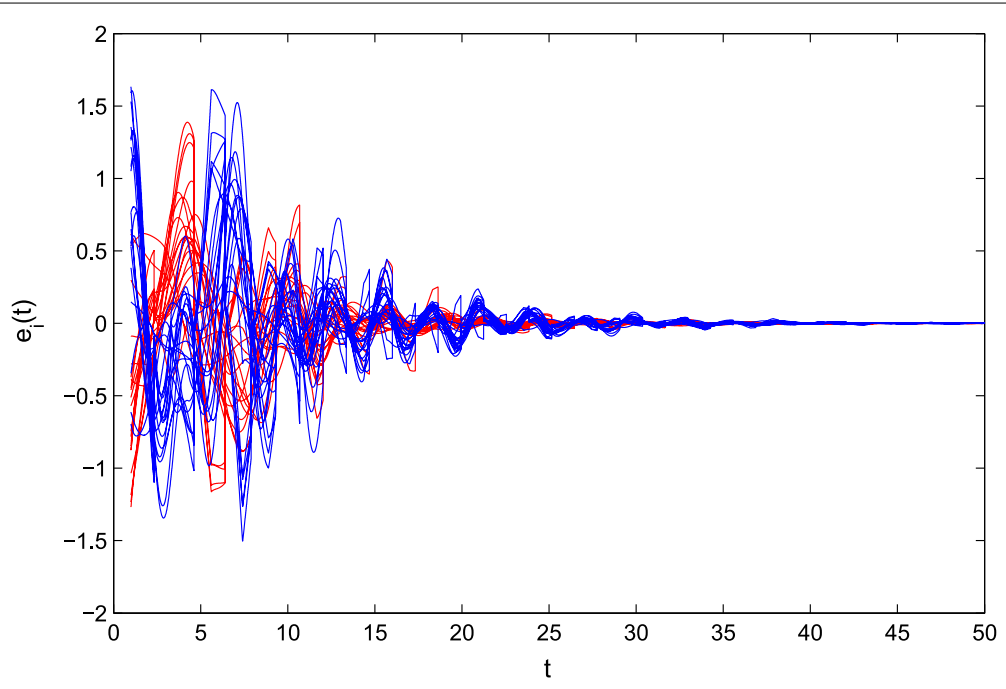

Figure 7 The orbits of $e_{i j}(t), i=1,2, \ldots, 20, j=1,2$. The red and blue lines denote the orbits of $e_{j 1}(t)$ and $e_{i 2}(t)$, respectively.

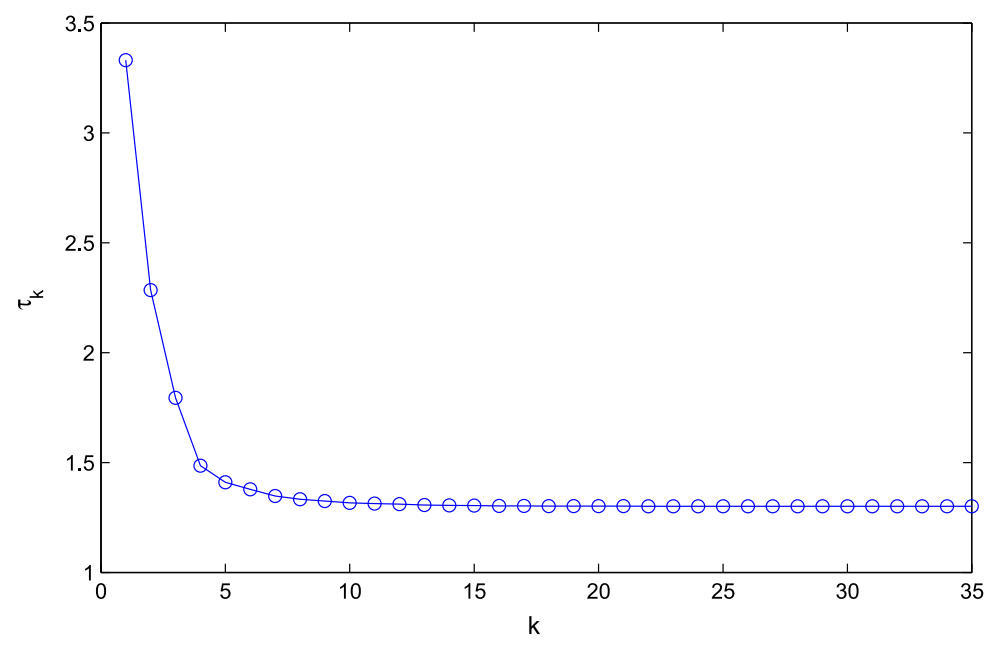

Figure 8 The orbit of $\tau_{k}$ vs $k$.

a sequence of maximum value problems. Noticeably, some constants with respect to the node dynamics and topology of network need not be calculated beforehand. Finally, the obtained results are verified to be correct and effective by performing several numerical simulations.

Competing interests

The authors declare that they have no competing interests.

Authors' contributions

All authors contributed equally to this work. They all read and approved the final version of the manuscript.

\section{Acknowledgements}

This work is jointly supported by the National Natural Science Foundation of China under Grant No. 61463022 and the Natural Science Foundation of Jiangxi Educational Committee under Grant No. GJJ14273. 
Received: 4 May 2015 Accepted: 14 July 2015 Published online: 06 August 2015

\section{References}

1. Boccaletti, S, Latora, V, Moreno, Y, Chavez, M, Hwang, DU: Complex networks: structure and dynamics. Phys. Rep. 424, 175-308 (2006)

2. Arenas, A, Díaz-Guilera, A, Kurths, J, Moreno, Y, Zhou, C: Synchronization in complex networks. Phys. Rep. 469, 93-153 (2008)

3. Chen, GR, Wang, XF, Li, X: Introduction to Complex Networks: Models, Structures and Dynamics. Higher Education Press, Beijing (2012)

4. Lu, JQ, Ho, DWC, Wu, LG: Exponential stabilization in switched stochastic dynamical networks. Nonlinearity 22 , 889-911 (2009)

5. Wu, XF: Complex projective synchronization in drive-response stochastic networks with switching topology and complex-variable systems. Adv. Differ. Equ. 2015, 129 (2015)

6. Wu, ZY, Fu, XC: Complex projective synchronization in drive-response networks coupled with complex-variable chaotic systems. Nonlinear Dyn. 72, 9-15 (2013)

7. Li, FF, Lu, XW: Complete synchronization of temporal Boolean networks. Neural Netw. 44, 72-77 (2013)

8. Rao, PC, Wu, ZY, Liu, M: Adaptive projective synchronization of dynamical networks with distributed time delays. Nonlinear Dyn. 67, 1729-1736 (2012)

9. Sun, W, Chen, Z, Kang, YH: Impulsive synchronization of a nonlinear coupled complex network with a delay node. Chin. Phys. B 21, 010504 (2012)

10. Yang, XS, Cao, JD, Lu, JQ: Exponential synchronization of uncertain delayed complex networks with nonidentical nodes and stochastic perturbations via hybrid adaptive and impulsive control. IEEE Trans. Circuits Syst. I, Regul. Pap. 59, 371-384 (2012)

11. Yang, XS, Cao, JD: Hybrid adaptive and impulsive synchronization of uncertain complex networks with delays and general uncertain perturbations. Appl. Math. Comput. 227, 480-493 (2014)

12. Deng, LP, Wu, ZY: Impulsive cluster synchronization in community network with nonidentical nodes. Commun. Theor. Phys. 58, 525-530 (2012)

13. Liu, DF, Wu, ZY, Ye, QL: Structure identification of an uncertain network coupled with complex-variable chaotic systems via adaptive impulsive control. Chin. Phys. B 23, 040504 (2014)

14. Liu, DF, Wu, ZY, Ye, QL: Adaptive impulsive synchronization of uncertain drive-response complex-variable chaotic systems. Nonlinear Dyn. 75, 209-216 (2014)

15. $\mathrm{Wu}, \mathrm{ZY}$, Liu, DF, Ye, QL: Pinning impulsive synchronization of complex-variable dynamical network. Commun. Nonlinear Sci. Numer. Simul. 20, 273-280 (2015)

16. Chen, YS, Hwang, RR, Chang, CC: Adaptive impulsive synchronization of uncertain chaotic systems. Phys. Lett. A 374, 2254-2258 (2010)

17. Lu, JQ, Wang, Z, Cao, JD, Ho, DWC, Kurths, J: Pinning impulsive stabilization of nonlinear dynamical networks with time-varying delay. Int. J. Bifurc. Chaos Appl. Sci. Eng. 22, 1250176 (2012)

18. Lu, JQ, Kurths, J, Cao, JD, Mahdavi, N, Huang, C: Synchronization control for nonlinear stochastic dynamical networks: pinning impulsive strategy. IEEE Trans. Neural Netw. Learn. Syst. 23, 285-292 (2012)

19. Lu, JQ, Ho, DWC, Cao, JD, Kurths, J: Single impulsive controller for globally exponential synchronization of dynamical networks. Nonlinear Anal., Real World Appl. 14, 581-593 (2013)

20. Wu, ZY: Adaptive impulsive outer synchronization between drive-response dynamical networks. Commun. Theor. Phys. 61, 590-594 (2014)

21. Sun, W, Lü, JH, Chen, SH, Yu, XH: Pinning impulsive control algorithms for complex network. Chaos 24, 013141 (2014)

22. Yu, WW, Chen, GR, Lü, JH, Kurths, J: Synchronization via pinning control on general complex networks. SIAM J. Control Optim. 51, 1395-1416 (2013)

23. Barabási, AL, Albert, R: Emergence of scaling in random networks. Science 286, 509-512 (1999)

24. Lorenz, EN: Deterministic nonperiodic flow. J. Atmos. Sci. 20, 130-141 (1963)

25. Hu, C, Yu, J, Jiang, HJ, Teng, ZD: Exponential stabilization and synchronization of neural networks with time-varying delays via periodically intermittent control. Nonlinearity 23, 2369-2391 (2010)

\section{Submit your manuscript to a SpringerOpen ${ }^{\ominus}$ journal and benefit from:}

$\checkmark$ Convenient online submission

Rigorous peer review

- Immediate publication on acceptance

- Open access: articles freely available online

- High visibility within the field

- Retaining the copyright to your article 